\title{
The role of topographic maps in river hydromorphology
}

\author{
Batuhan Kilic ${ }^{\mathrm{a}, *}$, Fatih Gulgen ${ }^{\mathrm{a}}$, Meltem Celen ${ }^{\mathrm{b}}$, Mehmet Salim Oncel ${ }^{\mathrm{c}}$, Halil Nurullah Oruc ${ }^{\mathrm{b}}$, \\ Sinem Vural ${ }^{\mathrm{c}}$ \\ ${ }^{a}$ Department of Geomatic Engineering, Yildiz Technical University, Istanbul, Turkey, batuhank@yildiz.edu.tr, fgulgen@yildiz.edu.tr \\ ${ }^{b}$ Earth and Marine Sciences Institute, Gebze Technical University, Kocaeli, Turkey, mkocal@gtu.edu.tr, horuc@gtu.edu.tr \\ ${ }^{c}$ Department of Environmental Engineering, Gebze Technical University, Kocaeli, Turkey, soncel@gtu.edu.tr, s.vural@gtu.edu.tr \\ * Corresponding author
}

Keywords: Water Framework Directive, River Hydromorphology, Topographic Maps, Reference Condition

\begin{abstract}
:
Hydromorphology is an umbrella discipline that examines and evaluates the physical, hydrological and morphological properties of water bodies and the processes underlying these properties. European Council (EC) has designed the water framework directive (WFD), one of the centerpiece documents of EC water legislation, to build sustainable water management (European Commission, 2000). Water quality, quantity, and water ecosystems are entirely interconnected. The hydromorphological data recorded to explain the state of the water source reveal the differences between assessment categories. The European Committee has put forward a detailed list of features recorded along the stream, riverbanks, riparian areas, and floodplains for standardization (CEN, 2002).
\end{abstract}

A proper understanding of the hydromorphological alterations in rivers through time is critical for scientists, policymakers, and managers conducting river management activities. The European countries have developed and used several hydromorphological monitoring and assessment methods with notable differences in aims, approaches, indicators, and collected data. Each technique provides descriptive information evaluating deviations from a natural state. Historical data, expert observations, modelling studies, and theoretical researches are used to reveal the natural form known as a reference condition of a water resource (Rinaldi et al., 2013). The reference condition that reflects the completely or rarely disturbed state must be explained to reveal the hydromorphological changes. Where field measurement data representing the reference condition are available, they should be considered first. However, it is difficult to perform field surveys due to their diverse characteristics and sizes in a high number of catchments. Therefore, the field survey is followed by historical topographic data sources, orthophotos and aerial photographs. Topographic maps are effective data sources to investigate river dynamics over a natural fluvial system spatially and temporally. In addition, official topographic maps at medium scale such as $1: 24 \mathrm{~K}$ or $1: 25 \mathrm{~K}$ have been produced and available for many countries for a long time. This study aims to reveal the hydro-morphological role of topographical maps that provide information about river geometry, land use, and artificial/natural features on the river, such as roads, buildings, lakes, or landforms. It was carried out using Turkish standard topographic maps of five different years between 1957 and 2018 at scale 1:25K. The selected study area is the Kocaeli-Gebze region in Turkey, which has been the center of industrialization and urbanization over the years. First, the factors affecting river hydromorphology in the study area were determined. Then, whether each of these factors can be obtained from topographic maps was examined one by one and features recorded along the river corridor were extracted. Approximately $82 \%$ of the recorded details and related reference conditions were obtained thanks to the spatial evaluations made for the selected study site using 1:25K topographic maps, which are more advantageous than time-consuming field studies and expensive satellite images. This result has shown that widely available current and old topographic datasets are an effective tool to reduce the number of field surveys conducted during hydromorphological studies.

\section{Acknowledgements}

This study has been financially supported by the Scientific and Technological Research Council of Turkey (TUBITAK) Project number 119 Y032.

\section{References}

CEN, 2002. A guidance standard for assessing the hydromorphological features of rivers. CEN TC 230/WG 2/TG 5:N32. 
European Commission, 2000. Directive 2000/60/EC of the European Parliament and of the Council of 23 October 2000 Establishing a Framework for Community Action in the Field of Water Policy. Official Journal L 327, 22/12/2000, Brussels.

Rinaldi, M., Belletti, B., Van de Bund, W., Bertoldi, W., Gurnell, A., Buijse, T. and Mosselman, E., 2013. Review on eco-hydromorphological methods. Deliverable 1.1, REFORM (REstoring rivers FOR effective catchment Management). In Project Funded by the European Commission within the 7th Framework Programme (2007-2013), Topic ENV. 2011.2. 1.2-1 Hydromorphology and Ecological Objectives of WFD, Grant Agreement 282656. 